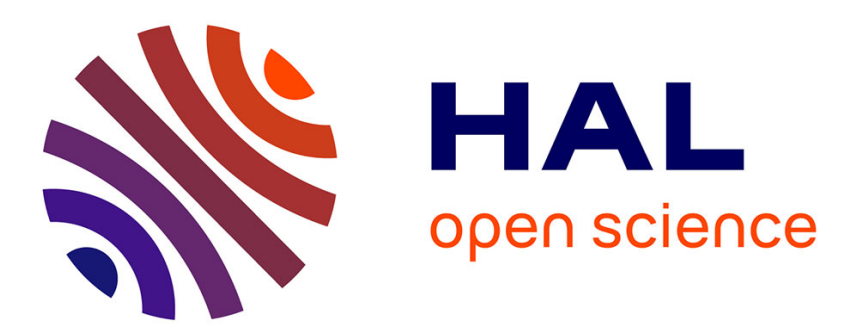

\title{
Comparison of WiMAX scheduling algorithms and proposals for the rtPS QoS class
}

Aymen Belghith, Loutfi Nuaymi

\section{To cite this version:}

Aymen Belghith, Loutfi Nuaymi. Comparison of WiMAX scheduling algorithms and proposals for the rtPS QoS class. EW 2008: European Wireless conference, Jun 2008, Prague, Czech Republic. hal-02316142

\section{HAL Id: hal-02316142 \\ https://hal.science/hal-02316142}

Submitted on 15 Oct 2019

HAL is a multi-disciplinary open access archive for the deposit and dissemination of scientific research documents, whether they are published or not. The documents may come from teaching and research institutions in France or abroad, or from public or private research centers.
L'archive ouverte pluridisciplinaire HAL, est destinée au dépôt et à la diffusion de documents scientifiques de niveau recherche, publiés ou non, émanant des établissements d'enseignement et de recherche français ou étrangers, des laboratoires publics ou privés. 


\section{Comparison of WiMAX scheduling algorithms and proposals for the rtPS QoS class}

\author{
Aymen Belghith \\ ENST Bretagne, France \\ 2 rue de la châtaigneraie, CS 17607, 35576 \\ Cesson-Sévigné Cedex - FRANCE \\ Email: aymen.belghith@enst-bretagne.fr
}

\author{
Loutfi Nuaymi \\ ENST Bretagne, France \\ 2 rue de la châtaigneraie, CS 17607, 35576 \\ Cesson-Sévigné Cedex - FRANCE \\ Email: loutfi.nuaymi@enst-bretagne.fr
}

\begin{abstract}
Scheduling algorithms are of utmost importance in WiMAX for efficient use of radio resources. A scheduling algorithm should take into account the WiMAX QoS classes and service requirements. It should also provide high throughput. In this paper, we propose a review of scheduling algorithms proposed for WiMAX. We focus on the real-time Polling Service (rtPS) QoS class. NS-2 simulations show interesting results. We highlight a problem that may exist with the WiMAX rtPS QoS class and we provide solutions for it. Simulation results concerning proposed WiMAX schedulers are discussed. We propose an enhancement of the maximum Signal-to-Interference Ratio (mSIR) scheduler, called modified maximum Signal-to-Interference Ratio (mmSIR). We show through extensive simulations that this enhancement provides better mean sojourn time in addition to an improvement in throughput.
\end{abstract}

\section{INTRODUCTION}

WiMAX technology based on the IEEE 802.16 standard [1], [2] has a very rich set of features. Indeed, it is a very promising Broadband Wireless Access (BWA) technology.

The objective is to have a highly efficient use of radio resources while transmitting different types of services. These services can have different constraints such as the traffic rate, maximum latency, and tolerated jitter. IEEE 802.16 power control and other capacity estimations were studied in [3].

The IEEE 802.16 Medium Access Control (MAC) specifies five types of QoS classes: Unsolicited Grant Service (UGS), real-time Polling Service (rtPS), extended real-time Polling Service (ertPS), non real-time Polling Service (nrtPS), and Best Effort (BE) QoS classes. UGS supports real-time service flows that have fixed-size data packets on a periodic basis. rtPS supports real-time service flows that generate variable data packets size on a periodic basis. ertPS is built on the efficiency of both UGS and rtPS. The BS provides unicast grants in an unsolicited manner like UGS. Whereas the UGS allocations are fixed in size, the ertPS allocations are dynamic. nrtPS is designed to support non real-time service flows that require variable size bursts on a regular basis. BE is used for best effort traffic where no throughput or delay guarantees are provided. Those service classes are defined in order to satisfy different types of Quality of Service (QoS) requirements. However, the IEEE 802.16 standard does not specify the scheduling algorithm to be used. Vendors and operators have to choose the scheduling algorithm(s) to be used. Three types of schedulers must be defined; an uplink and a downlink scheduler both in the Base Station (BS) and just an uplink scheduler for the Subscriber Station (SS) between the different simultaneous connections of the SS.

Some studies have been published about scheduling algorithms for WiMAX. Some of them address previously known schedulers evaluated for WiMAX such as in [4] and [5]. There are also schedulers specifically proposed for WiMAX [6][12]. These papers consider more or less complex details of the topic. Our paper focuses on the description of scheduling algorithms. We evaluate the performance of these schedulers used for rtPS QoS class and propose a simple enhancement which provides higher efficiency. An rtPS scheduler should manage radio resources as follows. The BS periodically provides unicast request opportunities in order to allow the SSs to specify their desired bandwidth allocations. When an SS receives an unicast request polling, it sends a bandwidth request. The bandwidth request contains the length of its uplink data connection queue.

The rest of paper is organized as follows. Section II gives an overview of the scheduling algorithms. Section III describes our design and implementation of some scheduling algorithms. Section IV presents the simulation results. Finally, concluding remarks and some further future work are made in Section V.

\section{WiMAX SCHEDUling AlgorithMS}

The WiMAX MAC layer is designed to support different types of applications and services having very different QoS requirements. The IEEE 802.16 standard does not specify the scheduling algorithm to be used. Vendors and operators have the choice among many existing scheduling techniques; they can also propose their own scheduling algorithms.

We can distinguish between two types of scheduling algorithms. The first type includes schedulers that are now wellknown. The second type represents the schedulers specifically proposed for WiMAX.

Many optimization criteria can be considered for scheduling algorithms such as the total maximum data rate, fairness, and operator revenue optimization. The description of some scheduling techniques is presented in the following. 


\section{A. Known Scheduling Algorithms}

In this section, we present some well-known schedulers. The simplest scheduling algorithm is the Round Robin (RR) scheduler. It distributes equal channel resources to all the SSs without any priority. The RR scheduler is simple and easy to implement. However, this technique is not suitable for systems with different levels of priority and systems with strongly varying sizes of traffic.

There is an extension of the RR scheduler, the Weighted Round Robin (WRR) scheduler, based on static weights. In the same context, we present the Deficit Round Robin (DRR) scheduler. The DRR scheduler associates a fixed quantum $\left(Q \_i\right)$ and a deficit counter $\left(D C \_i\right)$ with each flow $i$. At the start of each round and for each flow i, $D C \_i$ is incremented by $Q \_i$. The head of the queue Queue_i is eligible to be dequeued if $D C_{-} i$ is greater than the length of the packet waiting to be sent $\left(L_{-} i\right)$. In this case, $D C \_i$ is decremented by $L_{-} \mathrm{i}$. At each round, one packet at most can be sent (and then dequeued) for each flow.

Finally, we introduce the maximum Signal-to-Interference Ratio (mSIR) scheduler. It is based on the allocation of radio resources to subscriber stations which have the highest Signalto-Interference Ratio (SIR). This scheduler allows a highly efficient utilization of radio resources. However, with the mSIR scheduler, the users with a SIR that is always small may never be served.

\section{B. Scheduling Algorithms Proposed for WiMAX}

1) Temporary Removal Scheduler: The Temporary Removal Scheduler (TRS) scheduler [6] involves identifying the packet call power, depending on radio conditions, and then temporarily removing them from a scheduling list for a certain adjustable time period $T_{R}$. The scheduling list contains all the SSs that can be served at the next frame.

When $T_{R}$ expires, the temporarily removed packet is checked again. If an improvement is observed in the radio channel, the packet could be topped up in the scheduling list again, otherwise the process is repeated for another $T_{R}$ duration. In poor radio conditions, the whole process could be repeated up to $\mathrm{L}$ times at the end of which, the removed packed is added to the scheduling list, independently of the current radio channel condition.

2) Opportunistic Deficit Round Robin scheduler: The Opportunistic Deficit Round Robin (O-DRR) scheduler [7] is used in the uplink direction. The BS polls subscribers periodically. After each period, the BS determines the set of subscribers that are eligible to transmit and their bandwidth requirements. This set is defined as the eligible set. A number of conditions must be verified by an SS to be in this set:

- The queue is not empty.

- The received SIR is above a minimum threshold, denoted $S I R_{t h}$.

Once these conditions are satisfied, the subscriber is eligible to transmit during a given frame of the current scheduling epoch. The scheduled set changes dynamically depending on the wireless link state of subscribers. At the beginning of each scheduling epoch, the BS resets the eligible and scheduled sets and repeats the above mentioned process.

3) Frame Registry Tree scheduler: The Frame Registry Tree Scheduler (FRTS) scheduler [8] contains three operations: packet/request arrival, frame creation, and subscriber's modulation type change or connection QoS service change. The basic idea of the packet/request operation is to distribute packet transmissions in time frames, based on their deadline. For UGS and rtPS services, the packet deadline is equal to the arrival time plus the latency of this packet. The subtree of the last time frame where this packet can be transmitted is updated, if it exists. Otherwise, it is created. For nrtPS and BE services, the packet deadline does not need to be calculated. Then, the subtree of the last existing time frame is updated. The frame creation procedure decides on the frame contents. There are three cases:

- If the subtree of the first time frame contains a number of packets equal to one time frame, all these packets fill up the frame content.

- If the subtree of the first time frame contains a number of packets less than one time frame, the empty slots are occupied by packets from the next time frame subtrees and/or will be left for contention.

- If the subtree of the first time frame contains a number of packets more than one time frame, packets for BE service are moved to the next time frame subtree. If there are still excess packets to transmit, first nrtPS packets, then rtPS packets and finally UGS packets are deleted until the number of packets fit exactly into one time frame.

A change in a subscriber's modulation type or connection QoS service causes a moving of the corresponding subtree to the right modulation substructure or service substructure.

4) Adaptive rtPS scheduler: The adaptive rtPS scheduler [9] is used only for the rtPS QoS class. It is based on the prediction of the rtPS packets arrival. As defined in the IEEE 802.16 standard, the BS allocates bandwidth for rtPS traffic after receiving a bandwidth request. When the request is granted by the BS, the subscriber may receive from upper layers new rtPS packets. These packets will wait for the next grant to be sent and, therefore, suffer from extra delay. The basic idea of the adaptive rtPS scheduler is to propose an rtPS bandwidth request process in which the subscriber requests time slots for the data present in the rtPS queue and also for the data which will arrive. To estimate the data arrival, a stochastic prediction algorithm is defined in [9].

\section{Design AND Implementation OF SCHEDULing ALGORITHMS}

We have implemented a new Network Simulator (NS-2) WiMAX module and our module is based on the WiMAX NIST module [13] in order to implement and assess scheduling algorithms. The NIST module considers the Orthogonal Frequency Division Multiplexing (OFDM) physical layer of IEEE 802.16 and uses the Time Division Duplexing (TDD) technique. The existing implementation does not differentiate 
between the different service classes. The only scheduling algorithm implemented is described as follows. The BS leaves at least 10 symbols for the uplink direction and can use all the other symbols to send its packets in the downlink direction. Then, it allocates symbols to the ranging and bandwidth contentions and finally reserves all the remaining symbols for a single SS using the RR scheduler. Thus, at each frame, at most one subscriber can send its packets independently of its service class and its bandwidth request. The implemented scheduling algorithm has to be changed in order to differentiate between the service classes and take into account the QoS parameters. We added some QoS parameters to the existing implementation and we also used unicast and contention request opportunities as defined in the standard. For the scheduling mechanism, we implemented simple algorithms for UGS and BE. In this paper, we focus on rtPS schedulers and implement some of them in our NS-2 module.

\section{A. Design of Some Known Scheduling Algorithms}

In this section, we rapidly present the design of the RR, mSIR, and WRR schedulers. The RR scheduler performs as follows. At each frame, the BS determines the SS that will be served in a cyclic manner. The number of symbols to be allocated represents the minimum between the remaining symbols and the number of symbols that this SS has requested. If there are still remaining symbols, the BS serves the next SS until all the symbols are allocated.

The mSIR scheduler performs as follows. The BS sorts the SS according to their received SIR. The traffic that has the best SIR will be served first, and so on, until all the symbols are allocated.

Finally, the WRR algorithm performs as follows. According to the weights of the different SSs, the BS determines the SS that will be served. If there are still remaining symbols, the BS serves the next SS until all the symbols are allocated.

\section{B. Design of a Scheduling Algorithm Proposed for WiMAX}

In this section, we present our NS-2 design and implementation of the TRS scheduler. First, the BS determines the scheduling list. The scheduling list contains the SSs that can be scheduled in the current frame. Then, these SSs are served using another scheduling technique. Therefore, the TRS scheduler can be combined with a common scheduler like the RR and mSIR schedulers. The combined schedulers are called TRS+RR and TRS+mSIR schedulers, respectively.

If the RR scheduler is combined with the TRS scheduler and there are $k$ subscribers in the scheduling list, $1 / k$ of the whole radio resources is reserved to each subscriber.

If the mSIR scheduler is combined with the TRS scheduler, all the radio resources are reserved for the subscribers having the highest values of SIR.

\section{Design of Our Proposed Scheduling Algorithm}

In our implementation, the BS allocates symbols for the UGS, then rtPS and finally BE connections. For the rtPS

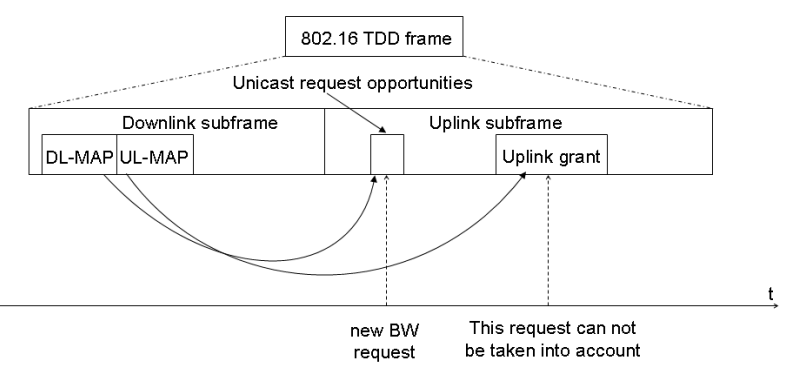

Fig. 1. Allocation of symbols for rtPS connection

connections, the BS allocates periodical unicast request opportunities and then, according to these requests, the symbols needed for the rtPS connections.

If the BS allocates unicast request opportunities and resource grants for rtPS connections in the same frame, the BS cannot immediately take into account the new length of the uplink data connection of the subscriber. The reason is that the BS allocates symbols for rtPS connections before receiving the latest unicast bandwidth request (see Fig. 1).

Moreover, the mSIR scheduler serves those subscribers having the highest SIR at each frame. So, subscribers having a slightly smaller SIR may be not served and then the mean delay to deliver data increases. We propose to modify the mSIR scheduler in order to decrease the mean time of sojourn. The mean sojourn time is the average time a data packet spends from its generation to its delivery at destination.

We propose that the BS only serves the subscribers that do not have unicast request opportunities in the same frame. The resulting scheduler is called the modified maximum Signal-toInterference Ratio (mmSIR) scheduler. The main steps of this proposed scheduler are shown in Fig. 2.

\section{Simulation Results}

\section{A. Simulation Model}

We have integrated the mSIR, mmSIR, WRR, TRS+RR, and TRS+mSIR schedulers into the NS-2 module. We have also integrated QoS parameters and added the UGS, rtPS, and $\mathrm{BE}$ service classes. The main parameters of the simulation are given in Table I. The sampling factor $(n)$ is equal to $144 / 125$ when the channel bandwidth is a multiple of 1.25 $\mathrm{MHz}$ (Frequency bandwidth chosen is $5 \mathrm{Mhz}$ ) and not a multiple of $1.75 \mathrm{MHz}$ (in this case, $n=8 / 7$ ) or $1.5 \mathrm{MHz}$ (in this case, $n=86 / 75$ ), see Table 213 of [1]. The possible values of the Cyclic Prefix are $1 / 4,1 / 8,1 / 16$, and $1 / 32$ (see Table 213 of [1]). The possible values of a frame duration are $2.0 \mathrm{~ms}, 2.5 \mathrm{~ms}, 4 \mathrm{~ms}, 5 \mathrm{~ms}, 8 \mathrm{~ms}, 10 \mathrm{~ms}, 12.5 \mathrm{~ms}$, and $20 \mathrm{~ms}$ (see Table 384a of [2]).

Since we are interested in comparing schedulers applied to rtPS connections, we consider different MCSs only for rtPS connections.

In our simulations, we have nine UGS and two BE connections using the QPSK1/2 MCS, and nine rtPS connections using different MCSs (QPSK1/2, QPSK3/4, 16QAM1/2, 16QAM3/4, 64QAM2/3, or 64QAM3/4). 


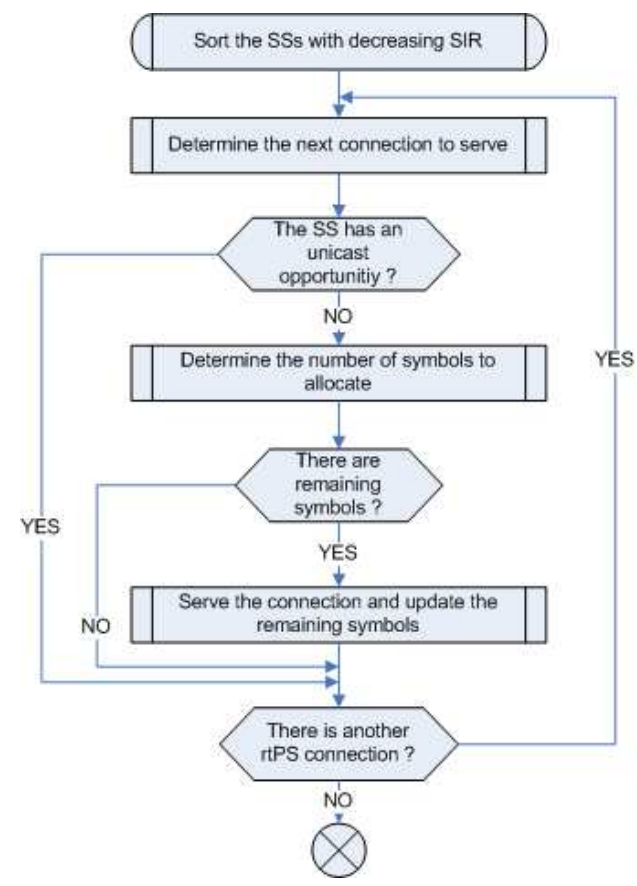

Fig. 2. Main steps of the proposed mmSIR scheduler

TABLE I

Main Parameters of the Simulation Model

\begin{tabular}{|c|c|}
\hline Parameters & Values \\
\hline \hline Frequency bandwidth & $5 \mathrm{MHz}$ \\
\hline Sampling factor (n) & $144 / 125$ \\
\hline Cyclic prefix (CP) & 0.25 \\
\hline Frame duration & $20 \mathrm{~ms}$ \\
\hline Mean packet length & $1024 b y t e s$ \\
\hline Simulation duration & $100 \mathrm{~s}$ \\
\hline Propagation model & Two Ray Ground \\
\hline Antenna model & Omni antenna \\
\hline Antenna height & $1.5 \mathrm{~m}$ \\
\hline Transmit antenna gain & 1 \\
\hline Receive antenna gain & 1 \\
\hline System loss factor & 1 \\
\hline Transmit power & 0.025 \\
\hline Receive power threshold & $205 \mathrm{e}-12$ \\
\hline Carrier sense power threshold & $0.9 *$ Receive power threshold \\
\hline Link adaptation & Enabled \\
\hline
\end{tabular}

\section{B. Comparison between Some Scheduling Algorithms}

In this section, we compare five scheduling algorithms: the RR, mSIR, WRR, TRS+RR, and TRS+mSIR schedulers.

Fig. 3 shows the number of delivered data packets as a function of the traffic load submitted to the network. The data packets are generated by a streaming multimedia application. This figure shows the inefficiency of the RR scheduler as it delivers the lowest number of packets. This is due to the fact that the channel quality of the subscribers is not taken into consideration. On the other hand, the RR scheduler shares the radio resources equitably between all the SSs.

In the same figure, we observe that both the mSIR and TRS+mSIR schedulers have good performance and deliver the highest number of packets. Indeed, these schedulers favor the

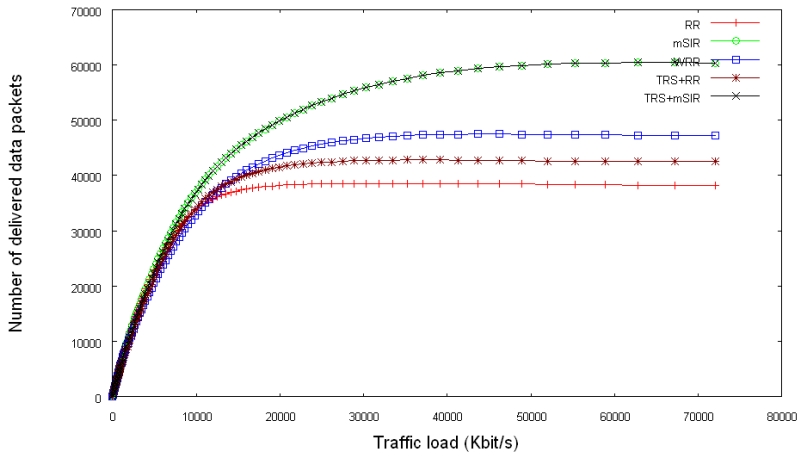

Fig. 3. Number of delivered data packets versus traffic load

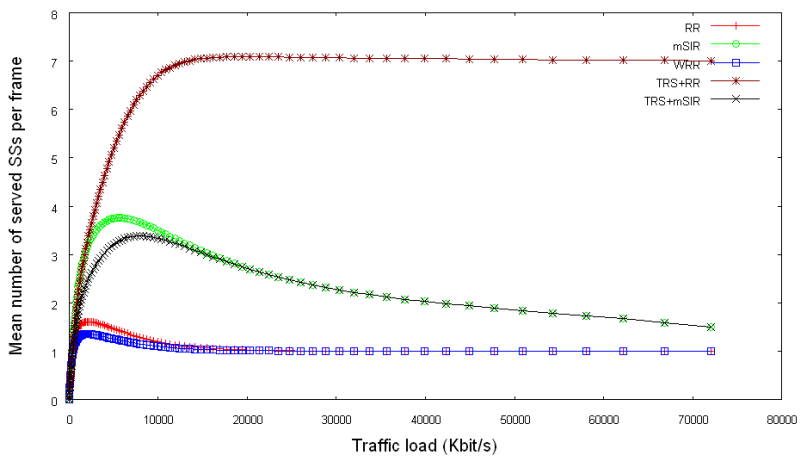

Fig. 4. Number of served SSs per frame versus traffic load

SSs having the highest SIR values and then using the most efficient MCSs.

We now consider the mean number of served SSs per frame. An SS is considered to be served when the BS allocates an uplink burst to this subscriber. Fig. 4 shows the mean number of served SSs per frame as a function of the data frame traffic load. The TRS+RR scheduler serves the highest number of SSs simultaneously in the same frame. Indeed, the TRS+RR scheduler serves all the SSs that belong to the scheduling list. In other words, the TRS+RR scheduler serves at least all the SSs having an SIR greater than a preset threshold.

We also observe that the RR and WRR schedulers only serve one SS per frame in medium and high traffic loads. This is because these schedulers may allocate the whole frame to one SS. Moreover, in medium and high loads, subscribers always have data packets to send.

The mean sojourn time as a function of traffic load is shown in Fig. 5. For real time applications such as Voice over Internet Protocol (VoIP) and Moving Picture Experts Group (MPEG), the mean sojourn time is a vital parameter. We note that the mSIR and TRS+mSIR require a large average delay to deliver a data frame. This is due to the freezing of traffic of SSs having a small SIR. We observe that the other three schedulers exhibit a much better mean sojourn time.

\section{Comparison between the mSIR and mmSIR Schedulers}

We recall that the ultimate purpose of our proposed mmSIR scheduler is to decrease the mean time of sojourn while not 


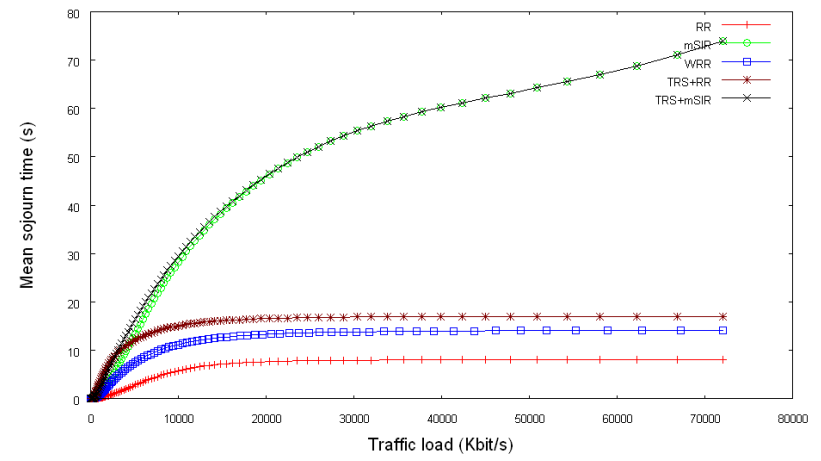

Fig. 5. Mean sojourn time versus traffic load

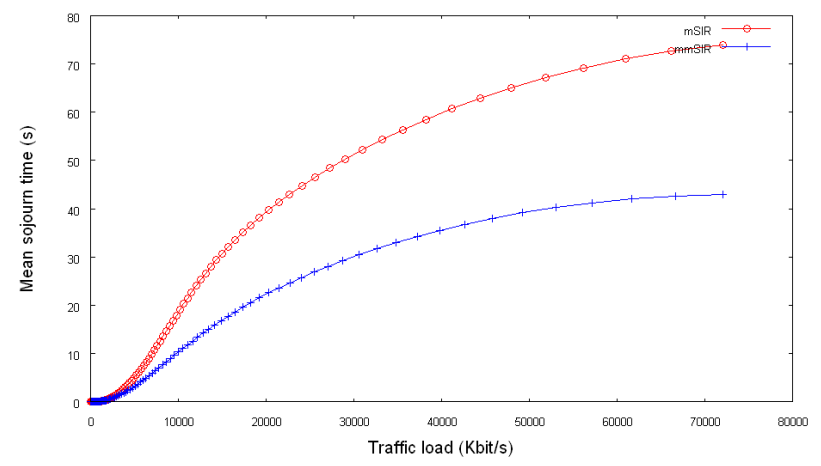

Fig. 6. Mean sojourn time versus traffic load

degrading the throughput with regard to the mSIR scheduler. Since the mSIR scheduler provides high throughput with a good SIR, we assume that the rtPS connections can use 16QAM2/3, 16QAM2/3, or 64QAM3/4. In this section, we compare the mSIR and mmSIR schedulers.

Fig. 6 shows the mean sojourn time as a function of traffic load. We verify that the mmSIR scheduler provides a decrease in the mean sojourn time. This is mainly due to the non freezing of the SSs having a small SIR. Indeed, the BS serves these SSs when it has already allocated unicast request opportunities to SSs having a higher SIR.

The number of delivered data packets as a function of traffic load is represented in Fig. 7. The mmSIR scheduler outperforms the mSIR scheduler. Indeed, the mmSIR scheduler, like the mSIR scheduler, favors those SSs having the highest SIR. If it does not serve an SS having unicast request opportunities, it gives priority to other SSs having higher SIR. Furthermore, the mSIR scheduler cannot immediately benefit from the unicast request opportunities of SSs since it has already reserved resources for rtPS connections before receiving the bandwidth requests. Moreover, the mmSIR scheduler serves fewer SSs than the mSIR scheduler (see Fig. 8). As a preamble is added to each uplink burst (see section 8.3.5.1 of [1]), the BS schedules more useful symbols when it serves fewer SSs per frame.

The mean number of served SSs per frame is represented in Fig. 8. The mSIR scheduler serves more SSs per frame because it always serves the SSs having the highest SIR. Then, these

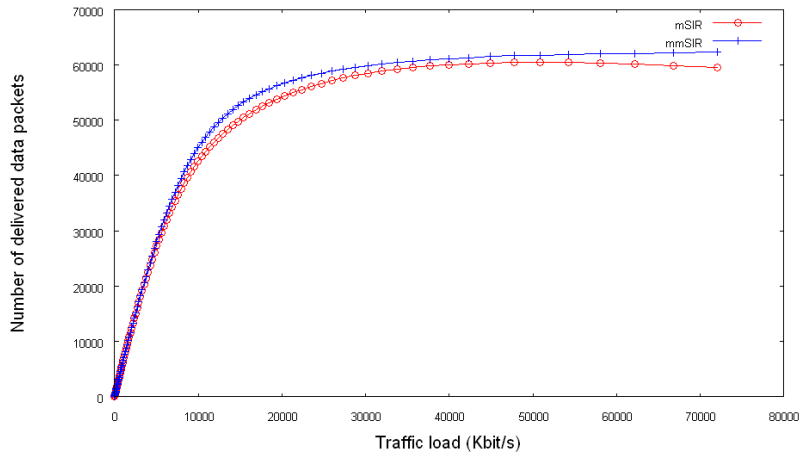

Fig. 7. Number of delivered data packets versus traffic load

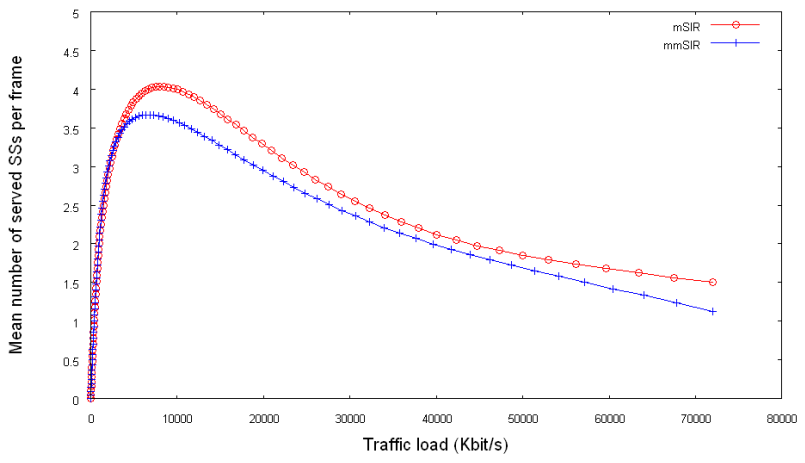

Fig. 8. Mean number of served SSs per frame versus traffic load

SSs usually do not have many data packets and share radio resources. On the other hand, the mmSIR scheduler does not serve an SS having unicast request opportunities. Thus, this SS will be served at the following frame. At this moment, it generates more data packets and its bandwidth request can be taken into account.

\section{CONCLUSION}

In this paper, we investigate the behavior of some WiMAX scheduling algorithms. We focus on the rtPS class. In our simulations, we verify that the mSIR and TRS+mSIR schedulers deliver the highest number of data packets, for a given scenario. Yet these algorithms require the highest delay to deliver these packets. The RR, WRR, and TRS+RR schedulers provide a better mean sojourn time while delivering fewer data packets than the mSIR and TRS+mSIR schedulers.

We highlight out a problem that can appear with the rtPS class and propose a solution to prevent this problem. This allows us to propose a more spectrum-efficient maximum SIR scheduler. We show through simulations that interesting improvements are obtained with the proposed mmSIR scheduler. This scheduler provides a much better mean sojourn time and also delivers more data packets with regard to the mSIR. A direction for future work is the study of the mmSIR for the OFDMA WiMAX PHYsical Layer. 


\section{REFERENCES}

[1] IEEE 802.16-2004, IEEE Standard for local and metropolitan area networks, Air Interface for Fixed Broadband Wireless Access Systems, Oct 2004.

[2] IEEE 802.16e, IEEE Standard for local and metropolitan area networks, Air Interface for Fixed Broadband Wireless Access Systems, Amendment 2: Physical and Medium Access Control Layers for Combined Fixed and Mobile Operation in Licensed Bands and Corrigendum 1, Feb 2006 (Approved: 7 Dec 2005).

[3] L. Nuaymi, Z. Noun, "Simple Capacity Estimations in WiMAX/802.16 System", the 17th Annual IEEE International Symposium on Personal Indoor and Mobile Radio Communications, PIMRC'2006, Helsenki, 11 - 14 September 2006.

[4] C.F. Ball, E. Humburg, K. Ivanov, and F. Treml, "Comparison of IEEE802.16 WiMAX Scenarios with Fixed and Mobile Subscribers in Tight Reuse", the 14th IST Mobile \& Wireless Communication Summit, Dresden, 19 - 23 June 2005.

[5] J. Chen, W. Jiao, and Q. Guo, "An Integrated QoS Control Architecture for IEEE 802.16 Broadband Wireless Access Systems", Global Telecommunications Conference, 2005, GLOBECOM'05, 28 November - 2 December 2005.

[6] C.F. Ball, F. Treml, X. Gaube, and A. Klein, "Performance Analysis of Temporary Removal Scheduling applied to mobile WiMAX Scenarios in Tight Frequency Reuse", the 16th Annual IEEE International Symposium on Personal Indoor and Mobile Radio Communications, PIMRC'2005, Berlin, 11 - 14 September 2005.

[7] H. K. Rath, A. Bhorkar, and V. Sharma, "An Opportunistic DRR (O-DRR) Uplink Scheduling Scheme for IEEE 802.16-based Broadband Wireless Networks", IETE, International Conference on Next Generation Networks (ICNGN), Mumbai, 9 February 2006.

[8] S. A. Xergias, N. Passas, and L. Marekos, "Flexible Resource Allocation in IEEE 802.16 Wireless Metropolitan Area Networks", the 14th IEEE Workshop on Local and Metropolitan Area Networks, LANMAN 2005, Chania, Greece, 18 - 21 September 2005.

[9] R. Mukul, P. Singh, D. Jayaram, D. Das, N. Sreenivasulu, K. Vinay, and A. Ramamoorthly, "An Adaptive Bandwidth Request Mechanism for QoS Enhancement in WiMax Real Time Communication", Wireless and Optical Communications Networks, 2006 IFIP International Conference on, Bangalore, India, 11 - 13 April 2006.

[10] Q. Liu, X. Wang, G. B. Giannakis, and A. Ramamoorthly, "A CrossLayer Scheduling Algorithm With QoS Support in Wireless Networks", IEEE Transactions on Vehicular Technology, 3 May 2006.

[11] T. Tsai, C. Jiang, and C. Wang, "CAC and Packet scheduling Using Token Bucket for IEEE 802.16 Networks", journal of communications, Vol. 1, No. 2, May 2006.

[12] K. Vinay, N. Sreenivasulu, D. Jayaram, and D. Das, "Performance Evaluation of End-to-end Delay by Hybrid Scheduling Algorithm for QoS in IEEE 802.16 Network", Wireless and Optical Communications Networks, 2006 IFIP International Conference on, 11 - 13 April 2006.

[13] Seamless and Secure Mobility: http://www.antd.nist.gov/seamlessandsecure.shtml, last visited in 15-012008. 